\title{
Flame deposition and characterization of large type IIA diamond single crystals
}

\author{
J. J. Schermer, W. J. P. van Enckevort and L. J. Giling \\ Research Institute for Materials, University of Nijmegen, Toernooiveld, 6525 ED Nijmegen (Netherlands)
}

\begin{abstract}
High quality diamond layers $70-400 \mu \mathrm{m}$ thick have been deposited homoepitaxially on $\{100\}$ and $\{110\}$ natural diamond substrates 3-4 $\mathrm{mm}$ in diameter by acetylene-oxygen flame deposition. For this purpose, a special substrate-cooling system was used in combination with a burner which had an orifice of $1.6 \mathrm{~mm}$. Microscopic observations revealed that the surface morphology (and thus the mechanism of diamond formation) is strongly dependent on the substrate orientation. Raman spectroscopy showed a significantly lower background fluorescence and a smaller full width at half-maximum (FWHM) of the diamond peak at $1332 \mathrm{~cm}^{-1}$ for layers grown on $\{100\}$ substrates than for those grown on $\{110\}$ substrates. Most crystals grown on $\{100\}$ substrates could be identified as type IIa diamond by IR absorption spectroscopy. However, small traces of nitrogen, not detectable by IR spectroscopy, could be detected by cathodoluminescence as nitrogen-vacancy pairs at $\lambda=575 \mathrm{~nm}$. The purest layers mainly exhibit blue band A luminescence. Finally, a large 0.4 carat single-crystal diamond was grown on top of a $\{110\}$ diamond substrate $6.5 \mathrm{~mm} \times 9 \mathrm{~mm}$. The layer is of type IIa but exhibits cracks parallel to the $\langle 112\rangle$ directions. Most other layers grown on $\{110\}$ substrates exhibit the same features, but none of the $\{100\}$ samples showed any sign of crack formation.
\end{abstract}

\section{Introduction}

In 1990, our group reported on the homoepitaxial growth of thick diamond films on $\{110\}$ seed crystals in an acetylene-oxygen flame at temperatures of approximately $900{ }^{\circ} \mathrm{C}[1,2]$. Homoepitaxial growth by this technique was confirmed by Snail and coworkers $[3,4]$, who reported growth rates up to $200 \mu \mathrm{m} \mathrm{h}^{-1}$ on $\{100\}$ substrates at high temperatures (over $1200^{\circ} \mathrm{C}$ ). Such high temperatures could not be achieved with our equipment and the reproducibility of the experiments appeared to be limited by poor temperature control.

Recently, however, a new improved temperaturecontrol system was developed in our group, allowing for high growth temperatures during long-term experiments. The process parameters using this set-up were optimized by means of polycrystalline diamond deposition on molybdenum substrates [5]. After these experiments, a number of macroscopically faceted single crystals were grown on circular $\{110\}$ and $\{100\}$ diamond substrates at temperatures up to $1320^{\circ} \mathrm{C}$. Several of these crystals revealed the occurrence of $\{113\}$ facets [6]. The torch used in these experiments had an orifice $1.0 \mathrm{~mm}$ in diameter. With this torch, deposits could be obtained which had a central area $3 \mathrm{~mm}$ in diameter, in which the morphology and thickness uniformity were more or less constant $[5,7]$. Outside this area, the deposits exhibited a poor quality or a significantly lower growth rate. Therefore, the dimensions of diamond substrates to grow well-faceted single crystals with this torch are limited to approximately $2 \mathrm{~mm}$ in diameter.

To obtain larger crystals, a torch with an orifice $1.6 \mathrm{~mm}$ in diameter was applied to the system. The quality of the large single crystals obtained using this upscaled flame is the subject of the present study.

\section{Crystal growth}

The experimental arrangement used in this study is almost identical to that described in detail in previous work [5]. The mass flows of the high purity source gases oxygen $(99.999 \%)$ and acetylene $(99.6 \%)$ are controlled to within \pm 0.2 standard litres per minute (slm). A commercially available acetylene-oxygen welding torch fitted with a tip with an orifice $1.6 \mathrm{~mm}$ in diameter is used to generate the flame. The reverse of a molybdenum substrate holder, fitted on top of a stainless steel cooler, is cooled by periodic jets of water from a vaporizer. The temperature in the holder is probed by a thermocouple and regulated by a temperature controller which changes the duty cycle of the water supply. The actual deposition temperature $T_{d}$ is measured to within $\pm 10^{\circ} \mathrm{C}$ relative $\left( \pm 20^{\circ} \mathrm{C}\right.$ absolute $)$ using a two-colour IR pyrometer. A cathetometer is employed to provide control of the distance $d$ between the inner flame cone and the substrate surface to within $\pm 0.1 \mathrm{~mm}$.

Polished natural type IIa diamond substrates with a 
surface roughness less than $20 \mathrm{~nm}$ have been used in all the experiments. Most of the substrates are circular with a diameter of 3 or $4 \mathrm{~mm}$ and a thickness between 140 and $520 \mu \mathrm{m}$. The orientation of the substrates is within $4^{\circ}$ of either $\{110\}$ or $\{100\}$. The substrates are soldered onto the substrate holder using a high temperature solder, ensuring a good thermal contact during deposition. The substrates are positioned in the acetylene feather along the central axis of the flame. Polycrystalline films deposited on molybdenum under similar conditions appear to have a central area which is homogeneous in quality and (within 10\%) in thickness. The diameter of this central area is approximately $7 \mathrm{~mm}$, so uniform growth conditions are ensured over the entire surface of the substrates.

The key parameters of the flame deposition process are considered to be the deposition temperature $T_{\mathrm{d}}$, the distance $d$ of the substrate from the inner flame cone, the absolute oxygen flow $f_{\text {ox }}$ and the acetylene supersaturation $S_{\mathrm{ac}}$ [5]. For a certain oxygen flow, $S_{\mathrm{ac}}$ is defined as the actual acetylene flow minus the acetylene flow of an ideal welding flame, divided by the latter. The ideal welding flame, for which the acetylene feather has just disappeared, is determined by sight. Previous work [5] clearly showed that both the growth rate and quality of the deposits increase with increasing $T_{\mathrm{d}}$ (also reported in refs. 8 and 9) and $f_{\text {ox }}$. Therefore, in this study, these parameters will be fixed to their maximum values $1200{ }^{\circ} \mathrm{C}$ and $3.4 \mathrm{slm}$, respectively, in all the experiments. The temperature is limited by the fact that the soldering material starts melting at higher temperatures [7], with the possible loss of thermal contact, temperature control and an expensive substrate. The oxygen flow is limited by the mass flow control system and the welding tip used. For the parameters $d$ and $S_{\mathrm{ac}}$, a compromise had to be found between the growth rate and homogeneity
TABLE 1. Range of deposition conditions

\begin{tabular}{ll}
\hline Welding torch & No. 3; orifice diameter, $1.6 \mathrm{~mm}$ \\
Substrate & Type IIa natural diamond \\
Deposition temperature $T_{\mathrm{d}}$ & $1200^{\circ} \mathrm{C}$ \\
Cone-to-substrate distance $d$ & $0.5-1.5 \mathrm{~mm}$ \\
Oxygene flow $f_{\mathrm{ox}}$ & 3.4 standard $1 \mathrm{~min}^{-1}$ \\
Acetylene supersaturation $S_{\mathrm{ac}}$ & $3.9 \% \cdot 5.4 \%$ \\
Deposition time $t_{\mathrm{d}}$ & $1.0-11.2 \mathrm{~h}$ \\
\hline
\end{tabular}

of the deposits. The range of the deposition conditions is given in Table 1.

\section{Characterization and discussion}

The deposited layers have been characterized while they were still attached to the natural diamond substrates. Therefore, the interpretation of the results obtained from Raman and IR spectroscopy is not completely unambiguous. However, all the substrates are of preselected type IIa diamond with well-known characteristics. Therefore, features different from those of natural type IIa crystals have to be assigned to the layers grown. Since the thickness of the deposits and the substrates are of the same order of magnitude, these features, if present, are most unlikely to be overlooked.

The variable deposition conditions and a number of characteristics of the samples grown are listed in Table 2. The samples grown on the different orientations are denoted by $\mathrm{D}$ (dodecahedral top face) and $\mathrm{C}$ (cubic top face). The thickness of the deposited layers $\left(h_{\mathrm{d}}\right)$ was obtained to within $\pm 5 \mu \mathrm{m}$ by subtracting the substrate thickness from the sample thickness, as measured by a micrometer.

The deposition rate $v_{\mathrm{d}}$ of layers grown on the $\{110\}$

TABLE 2. Variable conditions and results of the deposition experiments

\begin{tabular}{|c|c|c|c|c|c|c|c|c|c|}
\hline Sample & $\begin{array}{l}\text { Substrate } \\
(\mathrm{mm} \times \mu \mathrm{m})\end{array}$ & $\begin{array}{l}S_{\mathrm{ac}} \\
\pm 0.2 \\
(\%)\end{array}$ & $\begin{array}{l}d \\
\pm 0.1 \\
(\mathrm{~mm})\end{array}$ & $\begin{array}{l}h_{\mathrm{d}} \\
\pm 5 \\
(\mu \mathrm{m})\end{array}$ & $\begin{array}{l}v_{\mathrm{d}} \\
\pm 5 \\
\left(\mu \mathrm{m} \mathrm{h}^{-1}\right)\end{array}$ & $\begin{array}{l}n \\
\pm 10 \%\end{array}$ & $\begin{array}{l}\text { Peak } \\
\pm 0.2 \\
\left(\mathrm{~cm}^{-1}\right)\end{array}$ & $\begin{array}{l}\text { FWHM } \\
\pm 0.1 \\
\left(\mathrm{~cm}^{-1}\right)\end{array}$ & $\begin{array}{l}H_{\text {inc }} \\
\pm 20 \% \\
(\mathrm{ppm})\end{array}$ \\
\hline D1 & $\{110\} \phi 3 \times 318$ & 3.9 & 1.5 & 84 & 84 & 2.69 & 1332.6 & 4.2 & 1500 \\
\hline $\mathrm{D} 2$ & $\{110\} \phi 3 \times 309$ & 3.9 & 1.5 & 153 & 76 & 2.60 & 1331.5 & 3.9 & 1200 \\
\hline D3 & $\{110\} \phi 3 \times 259$ & 4.6 & 1.5 & 194 & 97 & 2.41 & 1332.1 & 3.4 & 900 \\
\hline D4 & $\{110\} \phi 3 \times 184$ & 4.6 & 1.0 & 266 & 133 & 2.55 & 1332.6 & 3.4 & 450 \\
\hline D5 & $\{110\} 6.5 \times 9 \times 235$ & $3.0-3.9$ & $1.0-1.5$ & $395-745$ & $35-66$ & - & 1332.5 & 3.2 & $<15$ \\
\hline $\mathrm{C} 1$ & $\{100\} \phi 3 \times 245$ & 3.9 & 1.5 & 138 & 69 & 2.64 & 1332.5 & 2.8 & $\ldots$ \\
\hline $\mathrm{C} 2$ & $\{100\} \phi 3 \times 244$ & 4.6 & 0.8 & 151 & 75 & 2.62 & 1332.6 & 2.9 & $-\ldots$ \\
\hline $\mathrm{C} 3$ & $\{100\} \phi 3 \times 262$ & 4.6 & 0.5 & 64 & 35 & 2.35 & 1332.0 & 2.9 & - \\
\hline $\mathrm{C} 4$ & $\{100\} \phi 4 \times 520$ & 4.6 & 0.6 & 205 & 66 & 2.67 & 1332.0 & 3.0 & 一 \\
\hline $\mathrm{C} 5$ & $\{100\} \square 4 \times 140$ & 4.6 & 0.8 & 145 & 73 & 2.44 & 1332.1 & 3.0 & - \\
\hline C6 & $\{100\} \phi 3 \times 311$ & 5.4 & 0.6 & 176 & 83 & 2.59 & 1332.0 & 2.5 & - \\
\hline $\mathrm{C} 7$ & $\{100\} \phi 4 \times 510$ & 5.4 & 0.7 & 370 & 111 & 2.60 & 1332.0 & 3.3 & - \\
\hline $\mathrm{C} 8$ & $\{100\}[4 \times 215$ & 5.4 & 0.7 & 515 & 123 & - & 1332.0 & 5.3 & - \\
\hline
\end{tabular}


substrates (D series) appears to increase with increasing $S_{\mathrm{ac}}$ (compare D1 and D2 with D3) and decreasing $d$ (compare D3 with D4). The same relation between $v_{\mathrm{d}}$ and these parameters was found for polycrystalline diamond deposition on molybdenum [5]. Layers grown on the $\{100\}$ substrates (C series) show a similar relationship between $v_{\mathrm{d}}$ and $S_{\mathrm{ac}}$ (compare C2-C5 with C6-C8). Although the relationship between $v_{\mathrm{d}}$ and $d$ of those layers is somewhat diffuse, it can be concluded that there exists an optimum growth rate at around $d=0.7 \mathrm{~mm}$. The refractive index $n$ of the layers grown was estimated from the ratio of the sample thickness to the optical path length, assuming a refractive index of 2.43 for the substrate. For most samples, the value of $n$ is comparable with that of natural diamond, within the experimental error $( \pm 10 \%)$.

The other features listed in Table 2 will be referred to in the next sections. The growth experiment from which sample D5 was obtained was somewhat different from the others and will be discussed separately.

\subsection{Microscopy}

The surfaces of the layers grown were investigated by optical differential interference contrast reflection (DICM) and scanning electron microscopy (SEM). Optical transmission and stress birefringence microscopy were employed to investigate the bulk of the layers.

Figure 1(a) shows that a number of flat facets have developed along the perimeter of the $\{110\}$ cylindrical seed crystals. These side faces, corresponding to the $\{111\},\{100\}$ and $\{113\}$ forms, have sharp boundaries with each other [6]. However, the $\{110\}$ top faces of these deposits have a cobbled structure, as can be seen in Fig. 1(b). This rough structure is expected from a $\mathrm{K} / \mathrm{S}$ face without the presence of extra surface periodic bond chains (PBCs), as a result of the adsorption of impurities or surface reconstruction [10]. Growth of these faces, which normally proceeds by the random addition of growth units at the surface [11], appears to be destabilized by the development of microfacets [12]. The average orientations of these facets were determined from sample D2-which shows the most developed microfacets using laser reflectometry with a wavelength beam of $632.8 \mathrm{~nm}$ from an $\mathrm{He}-\mathrm{Ne}$ laser.

By this technique, the orientations of the facets can be calculated from the angular distribution of back reflections of a parallel laser beam from the crystal surface. The reflected light from the $\{110\}$ sample shows an ovally shaped pattern, indicating the $\mathrm{mm}$ twodimensional point group symmetry of this face. This pattern originates from orientations close to the $\{h h k\}_{h<k}$ parts of the $\langle 110\rangle$ zone, as indicated by the grey area in the stereographic projection in Fig. 2. The most intense spots are reflected from orientations near $\{7713\}$
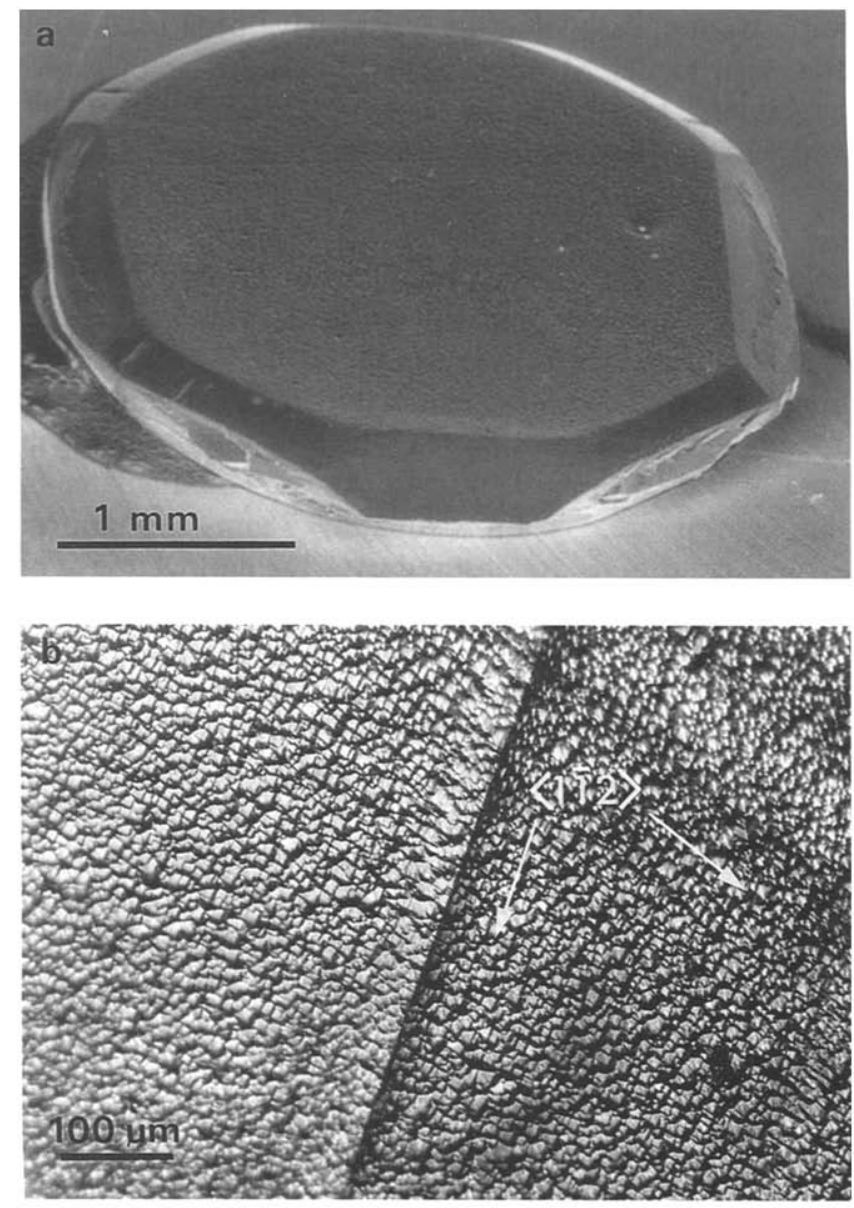

Fig. 1. Micrographs of a diamond single crystal (sample D4) grown on top of a $\{110\}$ natural diamond substrate. (a) SEM overview at an angle of about $45^{\circ}$ to the $\{110\}$ top face, viewing towards a $\{100\}$ side face. The $\{111\}$ side faces are located at the left- and right-hand sides of the sample, with the $\{113\}$ faces in between them and the $\{100\}$ face. (b) DICM detail of the cobbled $\{110\}$ top face structure, illuminated from the side to reveal the cracks.

and $\{119\}$, as indicated by the dark spots in the figure. For silicon, the stability of facets in the $\langle 110\rangle$ zone and the occurrence of $\{7713\}$ facets have been reported by Gardeniers et al. $[13,14]$. Most probably, the facets are somewhat rounded, which explains the discrepancy between the measured orientations and the $\langle 110\rangle$ zone. Orientations not corresponding to low index planes have also been found by an atomic force microscopy study of plasma-grown $\{110\}$ homoepitaxial diamond layers [15].

Most samples show crosswise cracks along the $\langle 1 \overline{1} 2\rangle$ directions in the $\{110\}$ surface (see Fig. 1(b)). The occurrence of cracks seems to be related to the lateral dimensions of the samples, because no cracking has been observed for smaller single crystals (1 $\mathrm{mm}$ in diameter) obtained by flame growth under similar conditions $[6,7]$. The fractures are most probably induced by the misfit stress resulting from a difference between the 


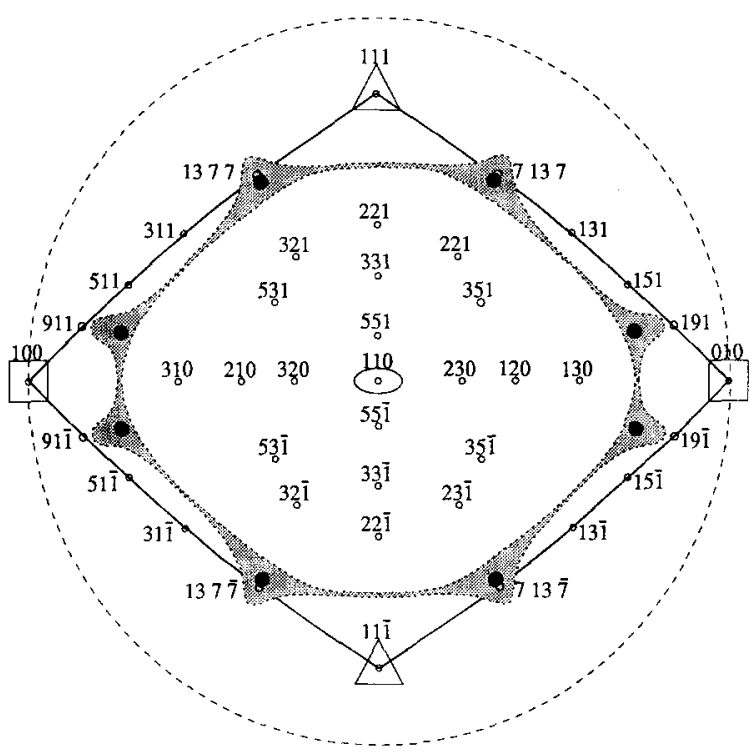

Fig. 2. Schematic drawing, in stereographic projection on the (110) plane, of the laser refections from the $\{110\}$ top face of sample D2 (grey area). The dark spots in the area indicate the orientations which gave the most intense reflections. All the orientations which can be revealed by laser reflectometry lay within $45^{\circ}$ from the central (110) orientation (broken line). The $\{h h k\}_{h \leqslant k}$ part of the $\langle 110\rangle$ zone is indicated by the full line.

lattice constants of the flame-deposited layers and the substrates $[2,16]$.

The strain in the deposited layers is likely to be released by cleavage along the $\{111\}$ planes, since these are the planes with the lowest cleavage energy [17]. Indeed, transmission optical microscopy reveals that the crack planes are parallel to the two $\{111\}$ planes perpendicular to the $\{110\}$ surface, and do not enter the substrate. No cleavage along the two other $\{111\}$ planes, which are not perpendicular to the $\{110\}$ surface, has been observed. This is explained by the fact that the tensile stresses resulting from lattice mismatch act parallel to the growth surface. In this case, $\{111\}$ cleavage cracks perpendicular to the $\{110\}$ surface are more easily formed than are those inclined to the surface, since the component of the tensile force perpendicular to the crack plane is a factor of $3^{1 / 2}$ larger in the first case. Because the stress can be released by the perpendicular cleavage planes in two directions parallel to the surface, there is no need for the other cleavage planes in the relaxation process.

A similar phenomenon was also observed for $\{110\}$ cleavage in f.c.c. fullerene crystals [18]. The $\{111\}$ side facets of all $\{110\}$ samples show cracks along the three $\langle 1 \overline{1} 0\rangle$ directions. Similar crack patterns were observed in $\{111\}$ epitaxial layers obtained by the hot filament technique $[19,20]$. In this case, all the possible $\{111\}$ cleavage planes make the same angle with the surface and, therefore, are equally effective for strain relaxation of this growth sector. From the fact that no relationship was observed between the surface morphology and fracture patterns, it is concluded that most cracking took place after the cessation of growth.

Optical birefringence microscopy reveals that the strain in the epitaxial layers is very localized and seems to be related to the cobbled surface structure [2]. The amount of stress could not be quantified, because the cobbled surface obstructed the measurements. Therefore, it was not possible to find a relationship between the occurrence of cracks and the amount of stress in the deposits.

The side faces of the samples grown on cylindrical $\{100\}$ substrates correspond to $\{111\}$ and $\{100\}$, as was also reported by Snail and coworkers [3,4], and give the crystals an octagonal shape (see Fig. 3(a)). The top faces of the samples are covered with macrosteps with flat regions in between, giving the unpolished samples a high optical transparency. According to a first-order $\mathrm{PBC}$ analysis, $\{100\}$ diamond is a rough $\mathrm{K} / \mathrm{S}$ face [21] without steps. As a result of a $(2 \times 1)$ surface reconstruction $[10,22-24]$ in which extra surface dimer bonds are created, a positive step free energy is obtained and the face appears like a flat $\mathrm{F}$ face. The thinner layers exhibit regions of parallel macrosteps (see Fig. 3(b)) with a step density and direction which differ from region to region.

Although the natural diamond substrates were flat with an overall orientation within $4^{\circ}$ from $\{100\}$, it is possible that they had mosaic regions with slightly different misorientations [25] which are reflected by the macrosteps. The thicker and faster grown samples reveal square step patterns (see Fig. 3(c)) with the macrosteps aligned along the $\langle 011\rangle$ directions, as is expected from the $(2 \times 1)$ surface reconstruction [10]. The square features become smaller and the surface roughness increases with the thickness of the samples. The surface of the thickest sample (C8) has essentially become highly textured polycrystalline in nature (see Fig. 3(d)). A similar roughening effect was also observed for thick samples grown on $\{110\}$ substrates [7].

Macrosteps have been observed for all the $\{100\}$ samples. Close examination of these steps by high magnification DICM shows that they are a few micrometres wide and are inclined at between $5^{\circ}$ and $20^{\circ}$ with respect to $\{100\}$. This was confirmed by laser reflectometry, where the most intense part of the reflection pattern forms a cross with arms along the $\langle 011\rangle$ directions.

Polarization microscopy showed that the strain in the deposited layers has a clear relationship with the $\{100\}$ surface structures: regions of stress are observed with striations perpendicular to the average step direction at the surface (see Fig. 4). This strain increases with the thickness of the grown layer, as is to be expected, and becomes localized in smaller domains which are reflected by the surface structure. It is possible that the surface 

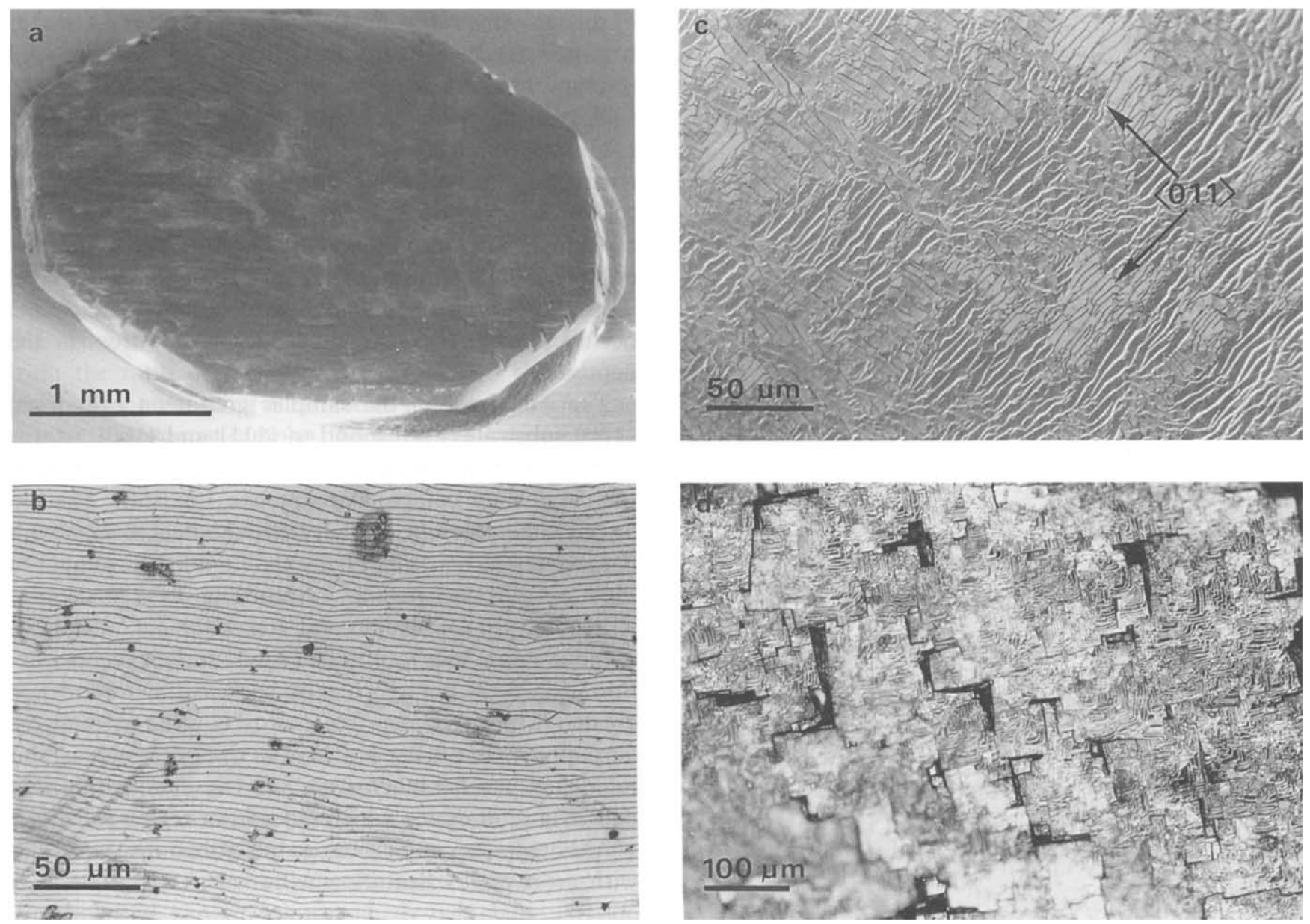

Fig. 3. Micrographs of diamond single crystals grown on top of a $\{100\}$ natural diamond substrate. (a) SEM overview of sample C1 at an angle of about $45^{\circ}$ to the $\{100\}$ top face. The $\{111\}$ side faces are located in front and at both sides of the sample, with the $\{100\}$ side faces in between them. DICM detail micrographs of $\{100\}$ top faces: (b) Region of parallel macrosteps on sample C1; (c) step patterns along the $\langle 011\rangle$ directions on sample C6; (d) the onset of polycrystalline deposition; square columns with growth spirals separated by deep (more than $10 \mu \mathrm{m})$ trenches on sample $\mathrm{C} 8$.

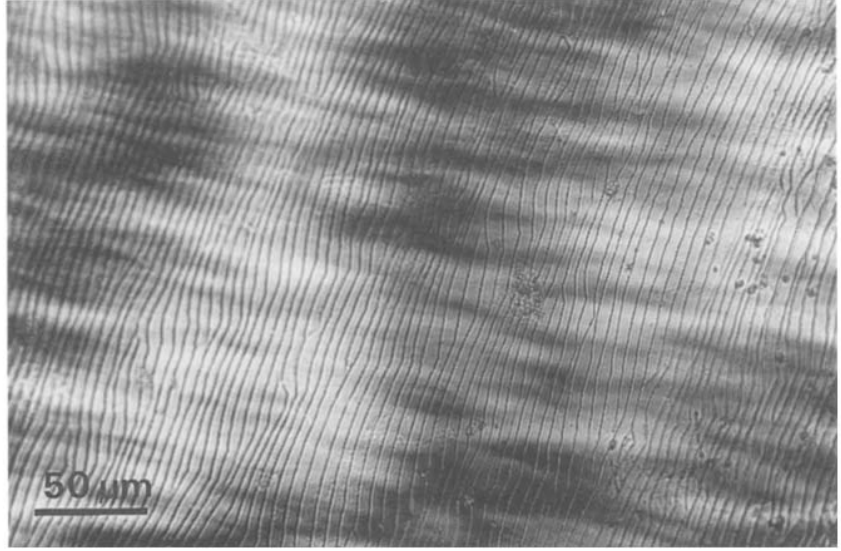

Fig. 4. Stress birefringence image of a flame-grown single-crystalline diamond layer (sample C1) with striations (horizontal) perpendicular to the average macrostep direction (vertical) at the surface. texture of sample $\mathrm{C} 8$ is a manifestation of strain release, although the exact mechanism is not known. Most layers are observed to have large stress patterns spreading out from step-emitting growth centres, which are related to lattice defects and diamond crystallites at the $\{100\}$ top face near the edges with the perpendicular $\{100\}$ side faces. It is possible that the surface reconstruction of both $\{100\}$ faces, which make an angle of $45^{\circ}$ with their common edge, plays a role in the formation of these defects. Although all the layers deposited on the $\{100\}$ substrates are strained, none of them is cracked.

From the above observations, it is concluded that, except for sample C8, all the layers grown are undoubtedly single crystalline in nature. The large differences between the layers grown on the $\{110\}$ substrates and $\{100\}$ substrates show that the mechanism of diamond formation is strongly orientation dependent and that the fingerprint of this mechanism is incorporated into the lattice as stress formations. 


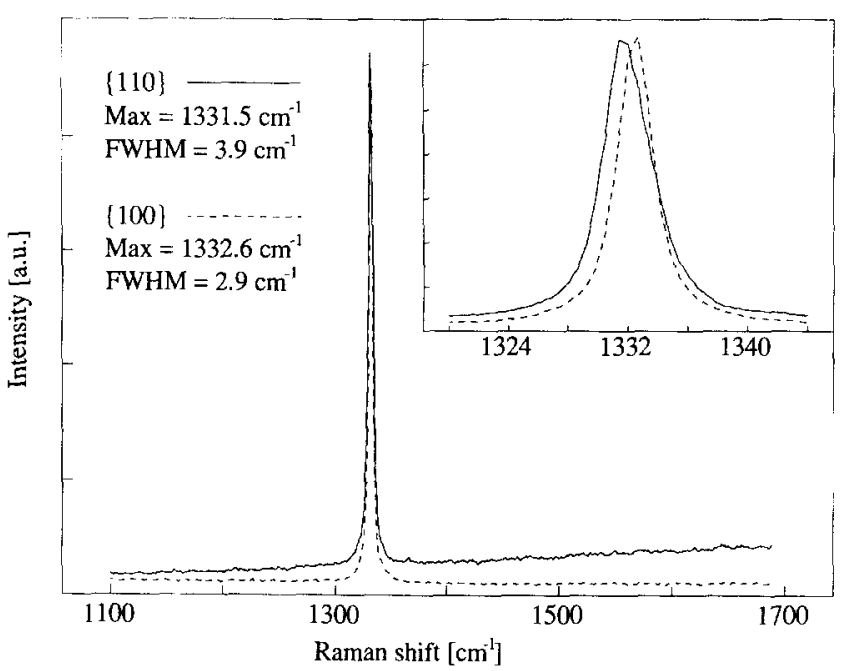

Fig. 5. Raman spectra of the single crystals D2 and C2. Inset: high dispersion spectra of the same samples.

\subsection{Raman spectroscopy}

The Raman spectra were recorded with a Dilor XY multichannel spectrometer using the $514.5 \mathrm{~nm}$ line of an $\mathrm{Ar}^{+}$laser as the excitation source. For all the samples, the position of the Raman peak and its full width at half-maximum (FWHM) are listed in Table 2.

The spectra of the $\{100\}$ samples differ significantly from the $\{110\}$ samples - the typical features of both are shown in Fig. 5. Except for samples $\mathrm{C} 7$ and $\mathrm{C} 8$, which have an enhanced surface roughness, the spectra of the samples with a cubic top face are indistinguishable from good quality type IIa natural diamond (FWHM of the diamond peak is $3 \mathrm{~cm}^{-1}$ or less). All the $\{110\}$ samples exhibit a higher background fluorescence, which increases at larger shifts, and a broader diamond peak ( $\mathrm{FWHM} \geqslant 3.4 \mathrm{~cm}^{-1}$ ).

Only sample D2 shows a small shift in peak position (see inset in Fig. 5) compared with the other samples and natural diamond $\left(1332.0-1332.5 \mathrm{~cm}^{-1}\right)$. The position of the diamond Raman peak is known to shift as a result of internal stresses [26, 27]. Possibly, sample D2 exhibits more stress in the layer, because it is the only $\{110\}$ sample which shows no sign of strain relief by cracking. As stated before, this could not be confirmed by stress birefringence, because of the cobbled surface structure of the sample.

The shift of the peak towards lower wavenumbers indicates that the stresses are tensile, which is in agreement with the smaller lattice constant of homoepitaxially grown single crystals compared with natural diamond, as reported by Janssen et al. [2]. A reduced lattice constant with respect to that of the natural diamond substrate could be introduced by a lower level of impurities or by an increased number of vacancies in the layers grown.

\subsection{IR spectroscopy}

The IR spectra were recorded using a Nicolet Fourier transform IR spectrophotometer. The transmission of all the $\{110\}$ samples monotonically decreases with increasing wavenumbers, owing to scattering losses at the rough surfaces of these samples (see dotted line in Fig. 6). This effect is strongest for samples which exhibit the most pronounced microfacets. $\mathrm{C} 7$ is the only $\{100\}$ single crystal which-albeit in a small extent-shows this effect $\left(65 \%\right.$ transmission at $800 \mathrm{~cm}^{-1}$ and $45 \%$ at $3200 \mathrm{~cm}^{-1}$ ).

Characteristic absorption spectra for both orientations are shown in Fig. 6. These absorption spectra were obtained from the single crystals with the largest grown layer-to-substrate thickness ratio (D4 and C5), and the $\{110\}$ spectrum is corrected for scattering losses. The $\{100\}$ samples show no features other than the intrinsic vibronic two-phonon band between 1700 and $2700 \mathrm{~cm}^{-1}$ [28], so are irrefutably identified as type IIa diamond. The small peaks between 1400 and $1800 \mathrm{~cm}^{-1}$ result from uncorrected background absorptions by water vapour in the spectrophotometer. Apart from the intrinsic diamond band, the $\{110\}$ samples also show a weak absorption band between 2800 and $3000 \mathrm{~cm}^{-1}$. In previously obtained single crystals grown at lower temperatures $\left(900^{\circ} \mathrm{C}\right)$, this band was much more pronounced and could be assigned to $\mathrm{C}-\mathrm{H}$ stretching of $\mathrm{sp}^{3}$ hybridized $\mathrm{CH}_{2}$ groups $[2,29]$.

A rough estimate of the amount $H_{\text {inc }}$ of hydrogen incorporated into the epilayer was obtained from the integrated absorption due to the band between 2700 and $3050 \mathrm{~cm}^{-1}$, and calibration with a polyethylene film [29]. The values of $H_{\text {inc }}$ for the $\{110\}$ samples were estimated in the same way and are listed in Table 2. These values are at least an order of magnitude lower

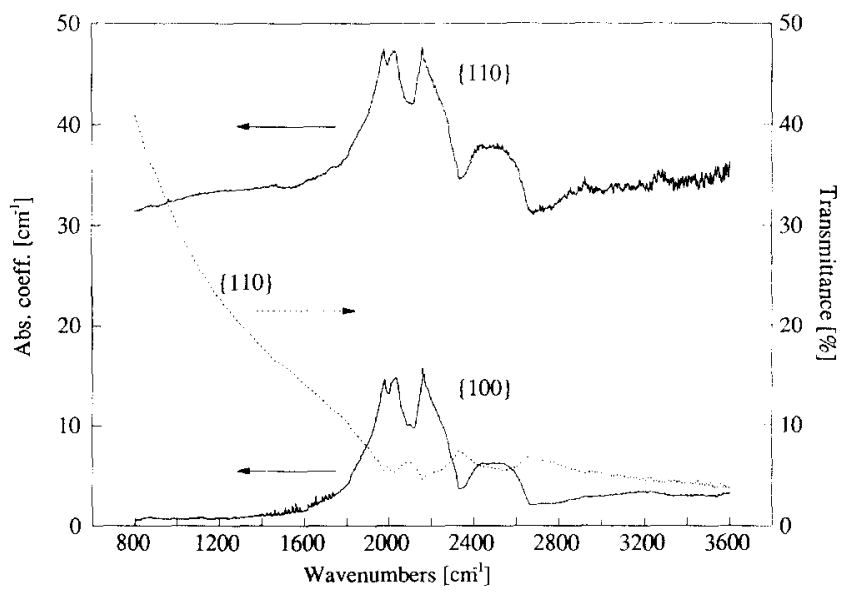

Fig. 6. IR transmission and absorption spectra of the diamond single crystal D4 and the absorption spectrum of sample C5. The absorption spectrum of D4 is corrected for scattering losses. The small peaks between 1400 and $1800 \mathrm{~cm}^{-1}$ result from uncorrected background absorptions by water vapour in the spectrophotometer. 
than those obtained for the samples grown previously $(1.5 \%-4.5 \%)$. From this, it can be concluded that the quality of the $\{110\}$ diamond layers is improved by high temperature deposition and are approaching type IIa diamond.

\subsection{Cathodoluminescence}

Since the penetration depth of the impinging electrons (typically $15 \mathrm{keV}$ ) is limited to a few micrometres [30], cathodoluminescence $(\mathrm{CL})$ is well suited to investigating the properties of the samples, because the signal obtained is strictly related to the grown layers. In this study, a commercially available 8200 MK II system for CL topography was used with a single monochromator system, in order to obtain room temperature spectra of the layers. The spectra are not corrected for the instrumental response, which is slightly better for higher photon energies. During examination, the crystals were pasted on to a copper block with heat-conductive cement to prevent heating up by the electron beam.

The full line in Fig. 7 shows the characteristic CL signal for the samples grown on the $\{110\}$ substrates. The orange-red $575 \mathrm{~nm}$ system originates from the $\{110\}$ top faces and has been attributed to nitrogen-vacancy pairs $[31,32]$. The blue band $\mathrm{A}$ emission, addressed to donor-acceptor pairs [33], dominates the luminescence from the $\{111\},\{113\}$ and $\{100\}$ side faces of the crystals. Blue band A luminescence from this $\{h h k\}_{h \leqslant k}$ part of the $\langle 110\rangle$ zone has also been reported for an epitaxial, flame-deposited diamond layer on a hemispherical diamond substrate [12]. It is suggested that the preferential nitrogen incorporation into the $\{110\}$ layers is related to the fact that the carbon atoms on

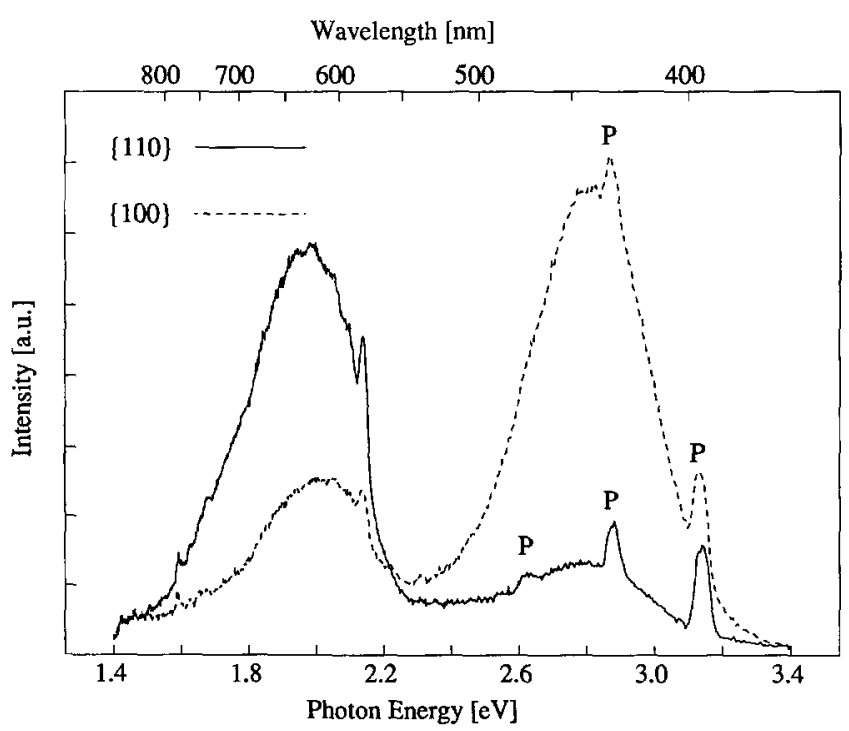

Fig. 7. CL spectra of flame-grown single-crystalline layers (samples D4 and $\mathrm{C} 7$ ). The plasma peaks produced in the $\mathrm{CL}$ unit are denoted by $\mathbf{P}$. this surface cannot form dimer bonds. This in contrast to faces in the $\langle 110\rangle$ zone mentioned, the surface atoms of which can dimerize [14]. However, the concentration of incorporated nitrogen is very low, since it could not be detected by IR spectroscopy.

The CL signal from the top face of the $\{100\}$ samples changes from orange-red for the thinnest and leaststepped surfaces to dark blue for the thickest and moststepped surfaces (see broken line in Fig. 7). The $\{111\}$ and $\{100\}$ side faces of all these samples only exhibit blue luminescence.

\section{Extended growth experiment}

From this work and previous investigations [7], there appears to be a correlation between the amount of surface roughness (at a micrometre scale) of the single crystals grown and the total deposition time. However, there are indications that "violent" growth conditions, i.e. high growth rates, are responsible for the enhanced surface roughness, which then becomes more profound after long deposition times.

To verify this hypothesis, a growth experiment lasting $11.2 \mathrm{~h}$ was performed, using a lean flame to suppress the growth rate. For this purpose, a large, somewhat irregularly shaped $\{110\}$ substrate of approximate dimensions $6.5 \mathrm{~mm} \times 9 \mathrm{~mm}$ was used. The position of the sample, initially $1.0 \mathrm{~mm}$ below the flame cone, was readjusted several times during the experiment, to correct for its increasing thickness during growth. The acetylene feather was observed to decrease during this long period of growth, so $S_{\text {ac }}$, initially set to $3 \%$, was increased whenever the feather became too small to cover the sample.

The experiment resulted in a total deposition of 0.4 carat, as determined by weight. The deposit is single crystalline in a central area approximately $7.5 \mathrm{~mm}$ in diameter (see Fig. 8(a)). To our knowledge, this is the largest reported single crystal obtained by flame deposition.

A small corner on the left-hand side of the sample, outside this region, is essentially polycrystalline. Except for this corner, the sample shows well-developed side faces, including $\{113\}$ facets. The layer grown exhibits a circular symmetric inhomogeneity in thickness, which varies from $750 \mu \mathrm{m}$ at the rim and the centre to $400 \mu \mathrm{m}$ in the area in between. The pitted structure at the righthand side of the layer was probably caused by etching during the short periods in which the substrate was not completely covered by the acetylene feather. Apart from these localized pits, the surface of the layer grown is significantly less rough than the other $\{110\}$ samples. This confirms that the surface roughness is caused by high growth rates rather than by long deposition times. 

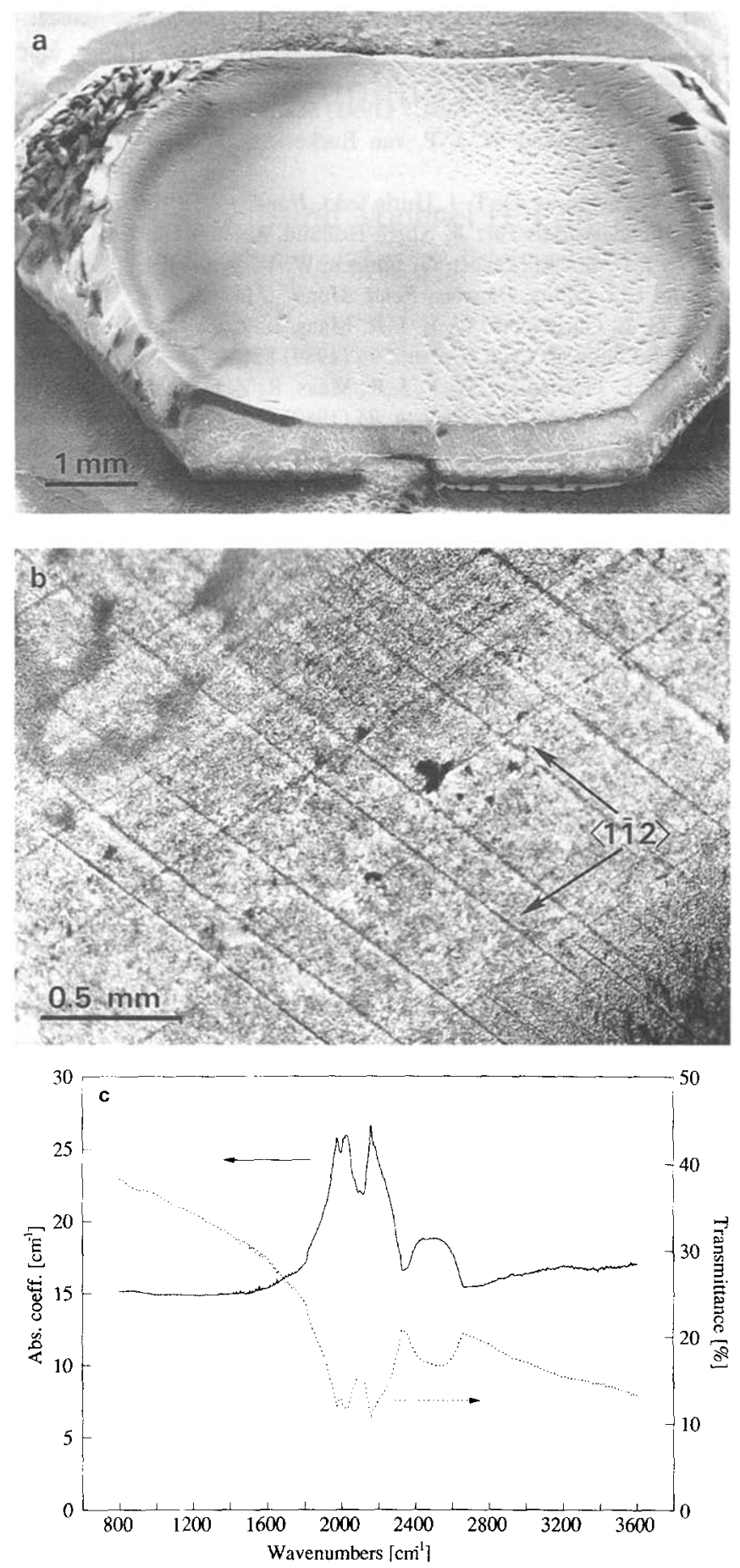

Fig. 8. (a) SEM overview of the flame-grown 0.4 carat single crystal (sample D5) and (b) a bright field transmission micrograph of the crack pattern in the $\{110\}$ top face of the specimen. (c) IR transmission and absorption spectra of the same layer. The absorption spectrum is corrected for scattering losses.

The layer grown exhibits an extended crack pattern with an average distance of a few hundred micrometres between adjacent parallel cleavage planes (see Fig. 8(b)). The increased density of the cracks compared with that in the other $\{110\}$ samples is related to the larger thickness of the layer and, presumably, also to the larger lateral dimensions. The Raman spectrum of the sample is almost identical to that of the $\{100\}$ samples and natural type IIa diamond, showing no increased background fluorescence and a reduced FWHM of the diamond peak compared with those for the other $\{110\}$ samples (see Table 2). The decreased surface roughness of the sample was also illustrated by the IR transmission spectrum, which is less affected by scattering losses than are the spectra of the other $\{110\}$ samples (compare the dotted lines in Figs. 6 and 8). The IR absorption spectrum of the sample corrected for scattering losses shows no significant amount of incorporated hydrogen or any other non-diamond features, so the grown layer is identified as type IIa diamond (see Fig. 8). In sharp contrast to the other $\{110\}$ samples, the $C L$ spectrum of the central part of the layer exhibits both the orange red $575 \mathrm{~nm}$ system and the blue band A luminescence.

\section{Conclusions}

Although the single crystals investigated in this study are still attached to their substrates, a large number of properties have been obtained from the samples, which are unmistakably related to the grown layer.

(i) Except for one, all the layers, $\{100\}$ as well as $\{110\}$, are single crystalline.

(ii) Large differences in the morphology between layers grown on the $\{110\}$ substrates and those on the $\{100\}$ substrates reveal that the mechanism of diamond formation is strongly orientation dependent.

(iii) Without exception, the layers exhibit stresses which show a clear relation with the surface structures of the samples. In contrast to the $\{100\}$ samples, the layers deposited on the $\{110\}$ substrates show strain release by cracking along $\{111\}$ planes perpendicular to the surface. The density of the cracks increases with the thickness of the layers and probably also with the deposition area.

(iv) Raman, IR and CL spectra of the $\{100\}$ samples are virtually identical to those of natural type IIa diamond and were significantly better than those obtained from the $\{110\}$ samples grown under similar conditions.

(v) A large $\{110\}$ single crystal with a deposited layer of 0.4 carat was obtained in a long deposition experiment with a lean flame and, therefore, low growth rate. This sample reveals that the surface roughness of the samples is caused by high growth rates rather than by long deposition times, and that type IIa diamond can be grown on $\{110\}$ substrates using a low supersaturation.

An attempt will be made to remove the substrates from some samples. This can be achieved by a complicated polishing technique, to minimize the risk of breaking the layer grown [7]. If one or more substrates can 
be removed successfully, additional characteristics of the remaining free-standing layers, such as UV-Vis spectra, will be reported in a forthcoming paper.

In this study, it has been demonstrated that large diamond single crystals with a high quality comparable with natural type IIa diamond can be grown by an acetylene-oxygen flame. Diamond deposition at temperatures above $1200{ }^{\circ} \mathrm{C}$ [3] or with turbulent flames [4, $34,35]$ seems to indicate that the quality of the deposits can still be improved. Therefore, it is plausible that synthetic crystals with a high crystalline perfection that surpasses that of natural diamond can be produced by flame deposition.

\section{Acknowledgments}

The authors wish to thank Drs. G. Janssen and Dr. $F$. Driessen for their invaluable help in recording the IR the CL spectra respectively. Dr. P. van Loosdrecht is acknowledged for the use of his Raman equipment. Furthermore, the authors are indebted to the diamond workers at Drukker International BV for the careful preparation of the substrates. This work was financially supported by The Netherlands Technology Foundation (STW) and the European Community Brite Euram Project BE 5099-92, under Contract BREZ-0147.

\section{References}

1 G. Janssen, W. J. P. van Enckevort, J. J. D. Schaminée, W. Vollenberg, L. J. Giling and M. Seal, J. Cryst. Growth, 104 (1990) 752.

2 G. Janssen, W. Vollenberg, L. J. Giling, W. J. P. van Enckevort, J. J. D. Schaminée and M. Seal, Surf. Coat. Technol., 47 (1990) 113.

3 K. A. Snail, C. L. Vold, C. M. Marks and J. A. Freitas, Jr., Diamond Relat. Mater., 1 (1992) 180.

4 K. A. Snail and L. M. Hanssen, J. Cryst. Growth, 112 (1991) 651.

5 J. J. Schermer, J. E. M. Hogenkamp, G. C. J. Otter, G. Janssen, W. J. P. van Enckevort and L. J. Giling, Diamond Relat. Mater., 2 (1993) 1149.

6 G. Janssen, J. J. Schermer, W. J. P. van Enckevort and L. J. Giling, J. Cryst. Growth, 125 (1992) 42.
7 J. J. Schermer et al., unpublished results, 1993.

8 R. Komanduri, K. S. Snail and L. L. Fehrenbacher, Philos. Mag. Lett., 62 (1990) 283.

9 K. V. Ravi, J. Mater. Res., 7 (1992) 384.

10 L. J. Giling and W. J. P. van Enckevort, Surf. Sci., 161 (1985) 567.

11 P. Bennema, in D. T. J. Hurle (ed.), Handbook of Crystal Growth 1, Fundamentals Part A, North-Holland, Amsterdam, 1993, Ch. 7.

12 W. J. P. van Enckevort, G. Janssen, W. Vollenberg, J. J. Schermer and L. J. Giling, Diamond Relat. Mater., 2 (1993) 997.

13 J. G. E. Gardeniers, W. E. J. R. Maas, R. Z. C. van Meerten and L. J. Giling, J. Cryst. Growth, 96 (1989) 821.

14 J. G. E. Gardeniers, W. E. J. R. Maas, R. Z. C. van Meerten and L. J. Giling, J. Cryst. Growth, 96 (1989) 832.

15 L. F. Sutcu, M. S. Thompson, C. J. Chu, R. H. Hauge, J. L. Margrave and M. P. D'Evelyn, Appl. Phys. Lett., 60 (1992) 1685.

16 L. M. Hanssen, W. A. Carrington, J. E. Butler and K. A. Snail, Mater. Lett., 7 (1988) 289.

17 J. E. Field, The Properties of Diamond, Academic Press, London, 1979.

18 M. Verheijen, W. J. P. van Enckevort and G. Meyer, Chem. Phys. Lett., 216 (1993) 72.

19 W. J. P. van Enckevort, G. Janssen and L. J. Giling, J. Cryst. Growth, 113 (1991) 295.

20 W. J. P. van Enckevort, G. Janssen, W. Vollenberg, M. Chermin, L. J. Giling and M. Seal, Surf. Coat. Technol., 47 (1991) 39.

21 P. Hartman, Z. Kristallogr., 121 (1965) 78.

22 W. S. Verwoerd, Surf. Sci., 103 (1981) 404.

23 T. Tsuno, T. Imay, Y. Nishibayashi, K. Hamada and N. Fujimori, Jpn. J. Appl. Phys., 30 (1991) 1063.

$24 \mathrm{~K}$. Okada, S. Komatsu, T. Ishigaki, S. Matsumoto and Y. Moriyoshi, J. Appl. Phys., 71 (1992) 4920.

25 P. L. Hanley, I. Kiflawi and A. R. Lang, Philos. Trans. R. Soc. London, Ser. A, 284 (1977) 329.

26 H. M. Grimsditch, E. Anastassakis and M. Cardona, Phys. Rev. B, 18 (2) (1978) 901.

27 M. Yoshikawa, G. Katagiri, H. Ishida, A. Ishitani, M. Ono and K. Matsumara, Appl. Phys. Lett., 55 (1989) 2608.

28 G. Davies, Chem. Phys. Carbon, 13 (1977) 1.

29 G. Janssen, W. J. P. van Enckevort, W. Vollenberg and L. J. Giling, Diamond Relat. Mater., 1 (1992) 789.

30 B. G. Yacobi and D. B. Holt, J. Appl. Phys., 59 (1986) R1.

31 L. H. Robins, L. P. Cook, E. N. Farabaugh and A. Feldman, Phys. Rev. B, 39 (1989) 13367.

32 A. T. Collins and S. C. Lawson, J. Phys: Condens. Matter, 1 (1989) 4029.

33 P. J. Dean, Phys. Rev., 139 (1965) A588.

34 P. Alers, W. Hänni and H. E. Hintermann, Diamond Relat, Mater., 2 (1993) 393.

35 K. A. Snail and C. J. Craigie, Appl. Phys. Lett., 58 (1991) 1875. 